Check for updates

Cite this: RSC Adv., 2017, 7, 24789

\title{
Fast detection of volatile organic compounds from Staphylococcal blood cultures by CDI-MS
}

Received 15th February 2017

Accepted 29th April 2017

DOI: $10.1039 / \mathrm{c} 7 \mathrm{ra01815k}$

rsc.li/rsc-advances

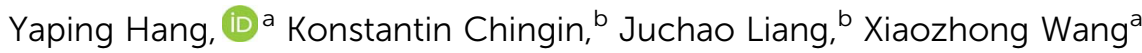 \\ and Longhua $\mathrm{Hu}^{\star \mathrm{a}}$
}

Rapid recognition of Staphylococcal bacteremia in humans is a serious challenge in clinical research. Here we report on direct analysis of molecular volatile organic compounds (VOCs) of blood cultures emitted by 35 patients with a suspicion of Staphylococcal bacteremia and 31 patients positively diagnosed with Staphylococcal bacteremia were fingerprinted by atmospheric corona discharge ionization mass spectrometry (CDI-MS) for the presence of four common Staphylococci (Staphylococcus aureus, S. hominis, S. haemolyticus, and S. epidermidis) which statistically account for ca. $90 \%$ of cases of Staphylococcal bacteremia in humans. All the infected blood cultures revealed characteristic and clearly distinct MS patterns specific to the presence of one of the four Staphylococci after an incubation time of 3-16 h. Technical replicates were incubated over 2-3 days for reference diagnosis using current conventional bacteremia detection. The results of our 6 month hospital study confirm peaks at $\mathrm{m} / \mathrm{z} 87$ (isobutyric acid) and $\mathrm{m} / \mathrm{z} 101$ (isovaleric acid) signals were specifically present in Staphylococci, and indicate that high suitability of CDI-MS for rapid, reliable and cost-efficient diagnosis of Staphylococcal bacteremia in humans.

\section{Introduction}

Bacteremia is one of the main causes of high morbidity and mortality worldwide, especially for neonate and children. Bacteremia cases in United States and California occurred at an average of 1.7 million hospitalizations per year. ${ }^{1}$ Morbidity and mortality rates of bacteremia are higher in China because of relatively weak domestic basic sanitation, lack of standardized antibiotic use, and low specimen submission. ${ }^{2-4}$ Staphylococci are extremely important pathogens of bacteremia. Coagulasenegative Staphylococci (CNS) are normal inhabitants of the human skin and mucous membranes. Proper acquisition of blood specimens seriously affects the accuracy of laboratory tests. Recently, CNS was recognized as etiologic agents of a wide variety of infections and was found to be the most frequent organisms isolated from blood cultures. ${ }^{4,5}$ Staphylococcus aureus (S. aureus), a major human pathogen, and resistant strains, especially meticillin-resistant $S$. aureus (MRSA), play important roles in nosocomial infection. Rapid detection of $S$. aureus and CNS in humans is a serious challenge in clinical research. ${ }^{5}$

The diagnostic "gold standard" of infectious disease is identification of bacterial pathogens. Note that traditional methods of bacterial identification are still mainly applied by

${ }^{a}$ Department of Clinical Laboratory, The Second Affiliated Hospital of Nanchang University, 1 Minde Road, Nanchang 330006, Jiangxi Province, P. R. China. E-mail: bingbinghang@hotmail.com; Tel: +86-791-86300410

${ }^{b}$ Jiangxi Key Laboratory for Mass Spectrometry and Instrumentation, East China Institute of Technology, Nanchang 330013, P. R. China majority of the clinical microbiology laboratories. The drawbacks of the current conventional identification methods highlight several unmet needs in Staphylococci diagnosis, such as necessity for accuracy and rapidity. As a result of these drawbacks, the processes of proliferation, smear microscopy, isolation, biochemical identification, and treatment are prolonged. Hence, rapid and accurate diagnosis could reduce the incidence of antibiotic abuses and unnecessary treatments.

Volatile organic compounds (VOCs) can be regarded as the "chemical information molecule" of bacteria. VOCs have great potential for development and wide application of as biomarkers for bacterial diagnosis. ${ }^{6,7}$ In this study, homemade and new high-sensitivity mass spectrometry (CDI-MS), followed by headspace analysis, was applied in the identification of common Staphylococci for early clinical diagnosis and treatment.

Other headspace-based chemical analytical methods can be used for the identification of volatile metabolites. However, these methods have drawbacks; chromatography demands higher operation and relatively complicated pre-treatment with poor practicability; ${ }^{\mathbf{2}}$ the electronic nose analysis is not highly sensitive and is not capable of analyzing chemical compositions ${ }^{9}$ the rising matrix-assisted laser desorption ionizationtime of flight-MS is inadequate for CNS identification, which is based on the difference of bacterial protein spectra, still requires bacteria detection after the isolation and culture; ${ }^{\mathbf{1 0}}$ and secondary electrospray ionization-MS also needs simple pretreatment with formic acid, with water used as an electrospray 
solution. ${ }^{\mathbf{1 1}}$ Various methods have significant shortcomings. ${ }^{\mathbf{1 2}}$ Previously Staphylococcal VOCs observed by mass spectrometry showed in Table $1,{ }^{13-15}$ bacteremia screening by ambient CDIMS, however, has obvious potential advantages: $:^{16-19}$ the speed of diagnosis ( $<1$ day) is higher than that by culture methods (total 2-3 days), greatly reducing the turnaround time for clinical testing; Another advantage of VOC analysis is its high throughput ( $c a$. 10-20 s per sample) because absolutely no sample pretreatment is necessary. CDI water vapor in the air with high ionization efficiency and without the use of highpressure gas, methanol, and other toxic reagents. Spectrum is relatively simple and easy to miniaturize. This work is an attempt to address the problems of time-consuming bacterial identification methods.

\section{Materials and methods}

\subsection{Sample ethical statement}

All experiments were performed in compliance with the guideline "Biomedical research ethics review method involving people" (China), and approved by the medical research ethics committee at the Second Affiliated Hospital of Nanchang University. Informed consents were obtained from human participants of this study.

\subsection{Conventional identification and preservation of pathogens}

Bacteria samples were isolated from patients in the Second Affiliated Hospital of NanChang University. Samples have previously been screened for the presence of bacteria. BacT/ ALERT 3D automated blood culture instrument (BD, Shanghai, China) was used to enrich the bacteria through phenotype screening of gram stain, oxidase, and $\mathrm{H}_{2} \mathrm{O}_{2}$ enzymes. The identification of clinical samples was carried out with VITEK2compact automated microbial analyzer (BioMerieux, Shanghai, China). When one of the four pathogens of interest ( $S$. aureus and CNS) was detected, the remainder of the sample was stored in frozen milk at $-80{ }^{\circ} \mathrm{C}$ (approximately $2 \mathrm{~mL}$ ) for further use.

\subsection{Preparation of the bacterial suspension}

Preserved strains or clinical isolates were recovered by culturing in blood agar plate at $35{ }^{\circ} \mathrm{C}$ overnight. The fresh single colony was inoculated in $0.45 \%$ sterile $\mathrm{NaCl}$ solution followed by dilution of bacterial suspension at different concentrations. Turbidity was determined with a turbidity meter (Vitek 2 DensiCHEK Plus, BioMerieux, Shanghai, China).

\subsection{Sample preparation}

2.4.1 Simulated blood culture. Negative blood cultures for reference measurements were selected based on the lack of bacteremia symptoms. Each negative blood culture was collected without splitting $(48 \mathrm{~mL})$ in glass bottles (Hemoline, BioMerieux, Shanghai, China), and $25 \mathrm{~mL}$ of liquid was removed and equally split into five fractions. Four out of the five fractions were respectively inoculated with $1 \mathrm{~mL}$ of $3 \times 10^{5} \mathrm{CFU}$ per $\mathrm{mL} S$. aureus, S. hominis, S. haemolyticus, and $S$. epidermidis suspension; the remaining fifth fraction was not inoculated. For the blank background reference, $1 \mathrm{~mL}$ of $0.45 \%$ sterile $\mathrm{NaCl}$ solution was used. All the fractions were incubated in $10 \mathrm{~mL}$ centrifuge tubes (Solarbio, Beijing, China). Patients with suspected bacteremia: $11 \mathrm{~mL}$ was removed from $48 \mathrm{~mL}$ of hemoline and was split into two fractions. The first $5 \mathrm{~mL}$ fraction was injected with $1 \mathrm{~mL}$ of $0.45 \%$ sterile $\mathrm{NaCl}$ solution, A second $6 \mathrm{~mL}$ fraction was incubated at the same condition for $16 \mathrm{~h} ; 5 \mathrm{~mL}$ of each fraction of simulated and suspected blood cultures was removed for MS analysis; the remaining $1 \mathrm{~mL}$ was used for biochemical identification. After incubation at $35{ }^{\circ} \mathrm{C}$ at different times, the time growth curves of VOCs were observed. $5 \mathrm{~mL}$ of confirmed bacteremia blood culture was directly detected by MS analysis.

\subsection{MS analysis of VOCs emitted by blood cultures}

2.5.1 Detecting condition. The indoor temperature was $20{ }^{\circ} \mathrm{C}$; humidity was $30 \%$; and nitrogen was used as the nebulizing gas at a flow rate of $3.2 \mathrm{~L} \mathrm{~s}^{-1}(0.1 \mathrm{MPa})$. Briefly, discharge needle was normally maintained at a high voltage $(+4 \mathrm{kV}$ in positive and $-3.5 \mathrm{kV}$ in negative ion detection mode) at ambient pressure. The angle between the needle and the outlet tubing was $30^{\circ}$. The distance from the tip of the needle to the end of the outlet tubing was $2 \mathrm{~mm}$. The distance from the tip of the needle to the inlet of the LTQ capillary was $6 \mathrm{~mm}$ as shown in Fig. 1.

2.5.2 Preparation of sample interface. For CDI-MS analysis, open centrifuge tube with a microbial culture was connected to the mounted cap. Changing the sample simply required disconnecting the tube and connecting a tube with the next sample to the same cap. This operational workflow allowed the high throughput and reproducibility of analysis. The headspace

Table 1 Staphylococcal VOCs previously observed by mass spectrometry

\begin{tabular}{|c|c|c|c|}
\hline Biomarker & Method & Specificity & Time (h) \\
\hline Isovaleric acid & GC-MS & Staphylococci & 25 \\
\hline 1-Pyrroline-5-carboxylate & LC-ESI MS/MS & S. aureus/S. epidermidis & $40-64$ \\
\hline Enolase & LC-ESI MS/MS & S. aureus/epidermidis & $40-64$ \\
\hline Catalase & LC-ESI MS/MS & S. aureus & $40-64$ \\
\hline Aconitate hydratase & LC-ESI MS/MS & S. epidermidis & $40-64$ \\
\hline$m / z 6230$ & MALDI-TOF MS & S. saprophyticus class A & 25 \\
\hline$m / z 3875$ & MALDI-TOF MS & S. aureus CC5 & 25 \\
\hline$m / z 6592$ & MALDI-TOF MS & S. aureus CC8 & 25 \\
\hline
\end{tabular}




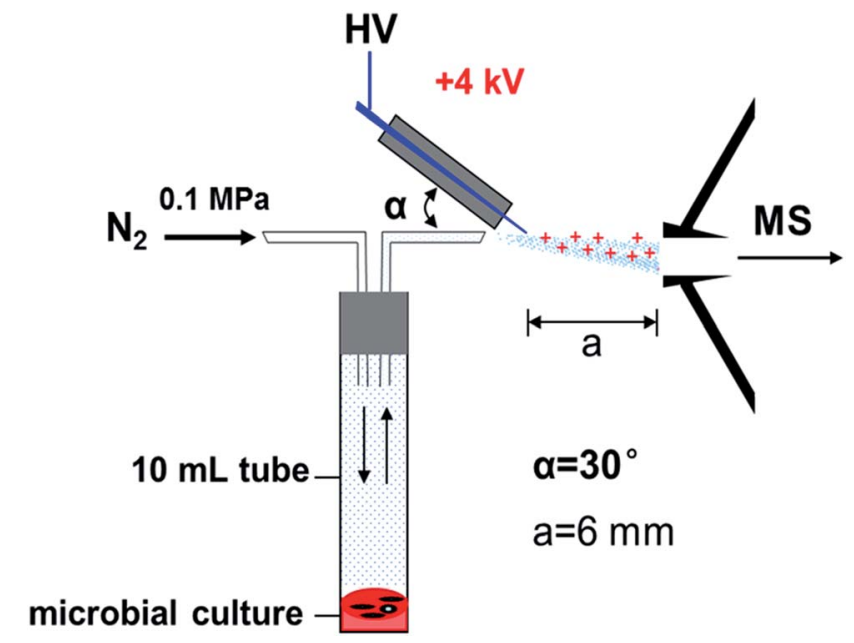

Fig. 1 Headspace VOC analysis of microbial cultures by CDI-MS.

VOCs of blood cultures were continuously transferred into the ionization region with nitrogen gas via plastic tubing. The assembly was firmly fixed in front of the instrument.

2.5.3 Detecting by CDI-MS. MS experiments were carried out on a commercial linear ion trap mass spectrometer (LTQXL, ThermoFischer, San Jose, CA, USA) interfaced with a homemade corona discharge-assisted extractive ionization source (SDAPCI), fully combining the advantages of various MS methods ${ }^{11,20,21}$ (schematically shown in Fig. 1). LTQ-Tune automatically optimized ion lens, detection systems, and other parameters. Analyses of the blank background reference and samples were done alternately. CDI-MS analysis of each replicate was obtained for $30-60 \mathrm{~s}$ in the $\mathrm{m} / \mathrm{z}$ range of $15-200$. The entire analysis sequence was independently repeated three times. Each fingerprint is averaged from the 120 scans obtained over the initial sampling period of $10 \mathrm{~s}$, when the headspace VOCs concentration is at a maximum. Chemicals identities of the sample were based on collision-induced dissociation (CID) experiments, CID analysis, up to $\mathrm{MS}^{3}$, with a collision energy (CE) of $10-30 \%$ and a mass-to-charge window width of 1.5 units, were performed on the characteristic ions for the sake of molecular structure identification.

\subsection{Data processing}

PCA was used to recognize difference/similarity between the microbial VOCs fingerprints based on the MS raw data by virtue of Matlab software (version 7.0, Mathworks, Inc., Natick, MA), respectively. Single, full-scan MS data was exported to Microsoft Excel as a one-to-one function. The file used the $\mathrm{m} / \mathrm{z}$ values as independent variables and the relative intensities of the fullscan MS as dependent variables. Hence, matrix was assembled of a total of MS and the total number of $\mathrm{m} / \mathrm{z}$ values collected in the MS. This matrix was readily loaded from the Excel file into the Matlab software for PCA analysis, respectively. The principle components for output were automatically determined by the Matlab software with the "princomp" function. The scores and loadings of the first two principle components (PCs) were 2D plotted in Matlab, and integral time of PCA was approximately several minutes.

\section{Results and discussion}

Previously, our team has analyzed the bacterial VOCs in vitro by ambient MS. ${ }^{18,19,22}$ In the present study, we extended the scope of the investigation and focused on clinical diagnosis of Staphylococcal bacteremia in humans. Over a period of six months, we detected blood cultures of 35 suspects and 31 positively diagnosed patients, including $9 S$. aureus isolates, $9 S$. hominis isolates, $6 \mathrm{~S}$. haemolyticus isolates, $7 \mathrm{~S}$. epidermidis isolates, and 19 simulated blood cultures. A total of 14 cases among 35 suspects were identified as $S$. aureus, and further research was needed for the other remaining pathogens. The full agreement between the results by the ambient MS analysis and the results by the conventional biochemical analysis demonstrated the potential of ambient MS for clinical applications.

First of all, the main VOC peaks of $S$. aureus were screened by positively diagnosed specimens. The baseline level in ion

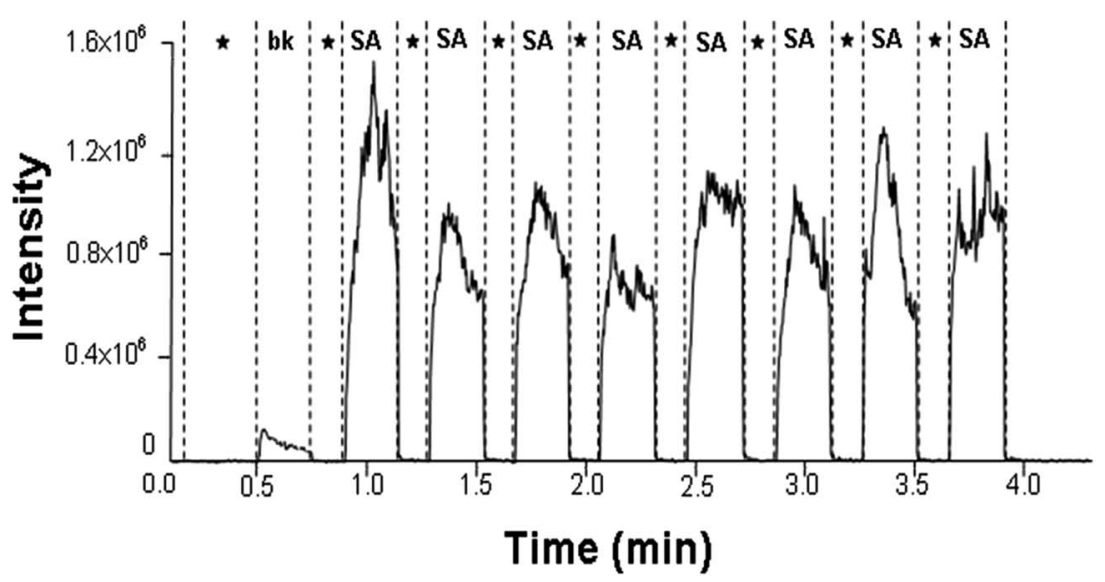

Fig. 2 Single ion chromatograms for $\mathrm{m} / \mathrm{z} 101$ signals in the CDI-screen of S. aureus. 8 strains was analyzed in succession. The samplings of ambient air are indicated with asterisk; bk: blank background reference; SA: S. aureus. 
chromatograms corresponds to the sampling of an empty centrifuge tube. Compared with the blank background reference, the signal intensity for $m / z 101$ observed was dramatically increased in the headspace of $S$. aureus blood cultures after culturing at $35{ }^{\circ} \mathrm{C}$ for $72 \mathrm{~h}$. The intensity increased up to the $10^{6}$ level (Fig. 2). The spike in the signal intensity at the beginning of each sampling in Fig. 2 can be attributed to the volatility of the ionized chemicals. Bacterial specific biomarker VOCs are critical for rapid pathogen identification based on $\mathrm{MS}^{7}$, but individual VOC is limited to the identification of a particular bacteria. Joint detection of multiple metabolites must be hastened to increase the sensitivity and specificity of VOC detection methods. $^{23}$ Fig. 3 displays typical CDI-VOC fingerprints in the main $m / z$ 60-200 range of simulated blood cultures inoculated with one of the four bacterial species ( $S$. aureus, S. haemolyticus, S. hominis, and $S$. epidermidis) at a concentration of $10^{4} \mathrm{CFU}$ per $\mathrm{mL}$ and grown aerobically at $35{ }^{\circ} \mathrm{C}$ for $16 \mathrm{~h}$. Peaks observed in the background-subtracted spectra do not necessarily belong to bacterial VOCs. Therefore, each mass spectrum was shown after the subtraction of signals from blank background reference from the same individual and incubated under the same conditions, all the signal to noise ratios of bacterial VOC fingerprints we detected were much higher than 3 after he subtraction of signals from blank background reference (e.g., signal to noise ratio of $m / z 101$ mainly were $195,168,206,225,251,201)$, it indicated that the

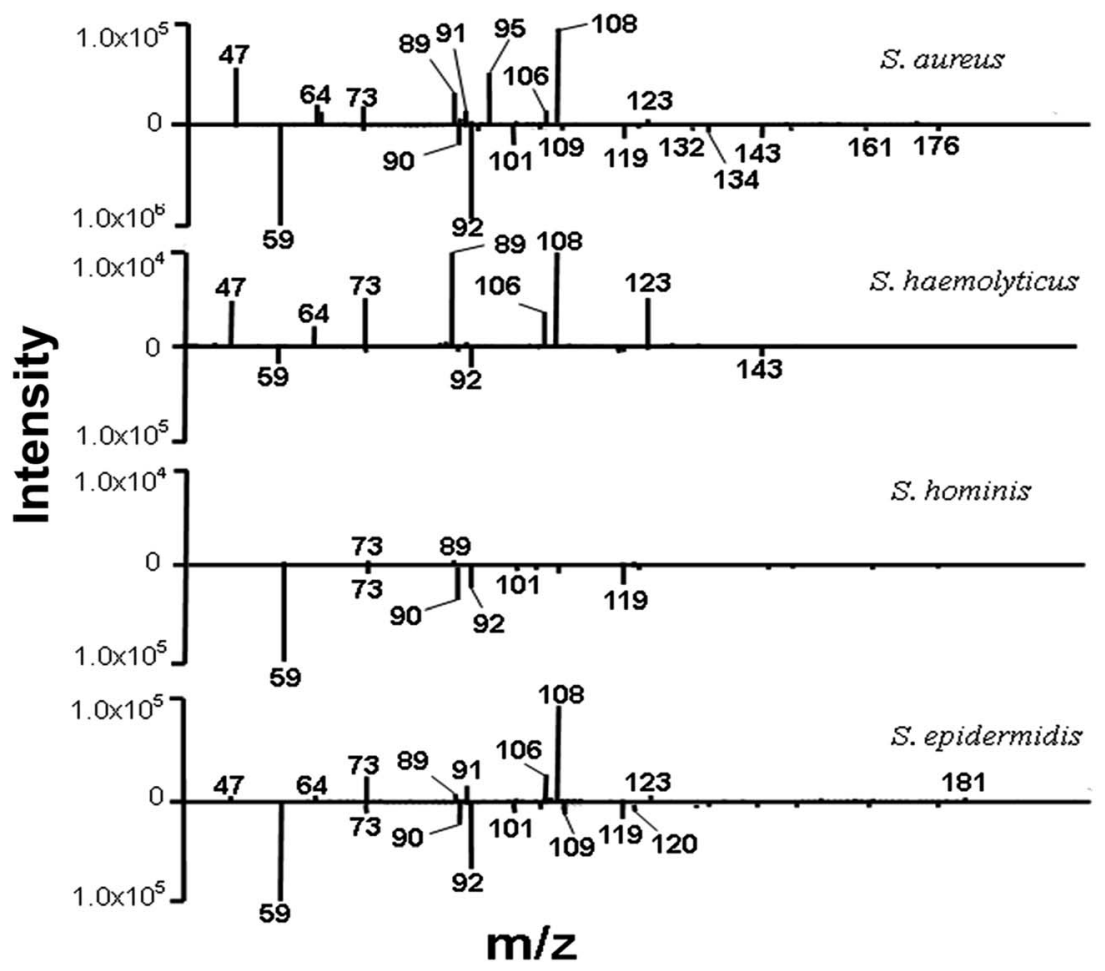

Fig. 3 Positive (top) and negative (bottom) ion mode VOC fingerprints of simulated blood cultures inoculated with one of the four Staphylococcal species.

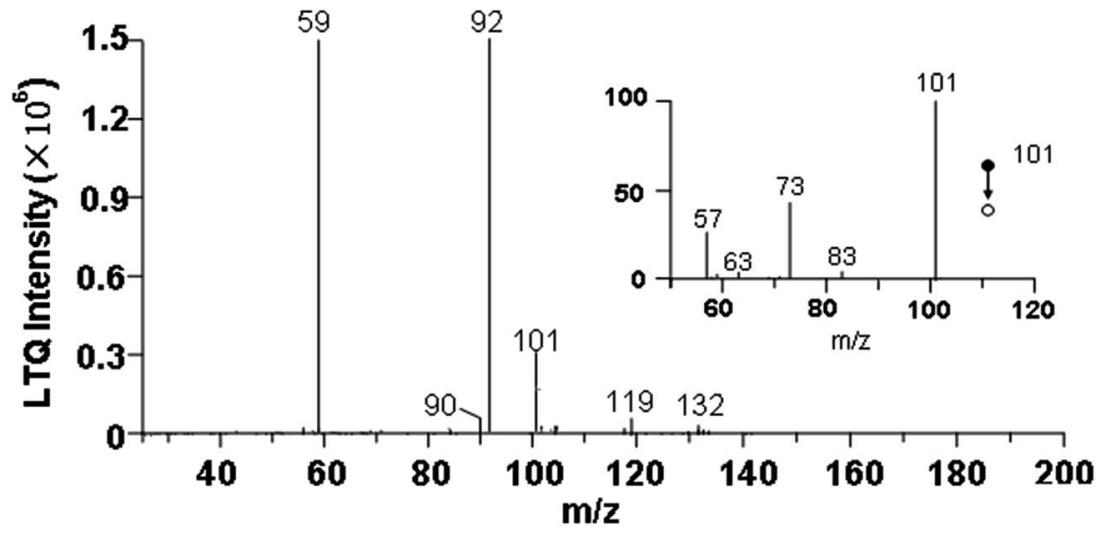

Fig. $4 \mathrm{CDI}$-MS spectrum of volatiles emitted by S. aureus culture after $16 \mathrm{~h}$ incubation, and MS/MS experiments of $\mathrm{m} / \mathrm{z} 101$. 
instrument was stable and sensitive to our requirements. A number of blood metabolic-related signals, e.g., $\mathrm{m} / z 59, \mathrm{~m} / z$ 90, and $m / z$ 92, are shared between different bacteria. Thus, the fingerprints can be readily distinguished by visual inspection. Separation of the bacterial metabolism associated with signals from the metabolism of blood was necessary to obtain the specific bacterial VOC fingerprints. ${ }^{24}$ The signals of $S$. aureus were relatively higher intensity. The signal intensities for $m / z 47$, $m / z 89, m / z 95$, and $m / z 108$ were dramatically increased, with the intensity reaching up to the $10^{5}$ level. Generally low signal peaks of $S$. haemolyticus and $S$. hominis were observed. By contrast, the signal peaks of $S$. epidermidis were higher than the other two CNS besides the common signal. Intensities of $m / z 73$, $m / z 106$, and $m / z 108$ were even higher than $S$. aureus, whereas the remaining signal peaks were low.

Based on the earlier published reports, the relative molecular mass of compounds corresponding to $\mathrm{m} / \mathrm{z}^{25}$ and the high specific signals were confirmed by MS/MS experiments (Fig. 4).

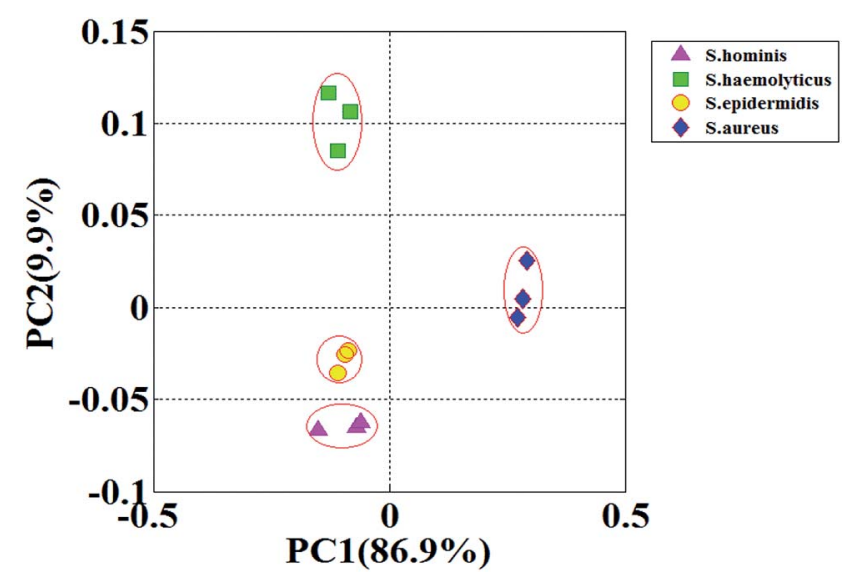

Fig. 5 PCA score plot of VOCs fingerprints from four simulated Staphylococci blood cultures in CDI-mode. Strains marked in different shapes or colors and the distance between each graph indicate the respective degree of difference; degree of dispersion between the same kinds of graphs indicates reproducibility.
We temporarily assigned some interesting signals in bacterial fingerprints to ethanol $\left(\mathrm{m} / z 47, \mathrm{CDI}^{+}\right)$, nitric acid $\left(\mathrm{m} / z 64, \mathrm{CDI}^{+}\right)$, phenol $\left(\mathrm{m} / z \mathrm{~g}, \mathrm{CDI}^{+}\right)$, benzaldehyde $\left(\mathrm{m} / z \mathrm{108}, \mathrm{CDI}^{+}\right)$, acetic acid $\left(m / z 59, \mathrm{CDI}^{-}\right)$, ammonium propionate $\left(\mathrm{m} / z\right.$ 90, $\left.\mathrm{CDI}^{-}\right)$, and aniline $\left(\mathrm{m} / \mathrm{z}\right.$ 92, $\left.\mathrm{CDI}^{-}\right)$. The biomarker signals were isovalerate $\left(\mathrm{m} / z 101, \mathrm{CDI}^{-}\right)$and isobutyric acid $\left(\mathrm{m} / z \mathrm{89}, \mathrm{CDI}^{+} ; \mathrm{m} / z \mathrm{87}, \mathrm{CDI}^{-}\right)$. Notably, $m / z 101$ signal specifically existed in all four Staphylococci at different intensities, thus helping to differentiate Staphylococci from the other bacterial species. Moreover, the presence of $\mathrm{m} / z 132$ and $\mathrm{m} / \mathrm{z} 134$ suggested that isovalerates are secondary metabolites of these two substances. All the features of Staphylococcal spectrum introduced above can be used for the identification of pathogens.

Handling multi-variable MS data by PCA has significant advantages. $^{26,27}$ The replicates of $S$. aureus are clustered at the largest distance from the rest of the bacterial strains; $S$. haemolyticus are distant from $S$. aureus with highest distinction; $S$. hominis and $S$. epidermidis are close to each other but the boundaries are clear (detailed in Fig. 5). Overall, the discrimination of VOC fingerprints from the four Staphylococci is satisfactory using PCA, especially for $S$. aureus and $S$. haemolyticus, which are indistinguishable by traditional methods because of the presence of hemolysis. Early exclusion of contamination with CNS will greatly reduce the abuse of antibiotics and reduce health care costs for patients.

Moreover, the incubation time of $16 \mathrm{~h}$ used in the above simulated experiment is substantially shorter than in traditional clinical routines (48-72 h), thereby allowing space for further improvement. Therefore, we also investigated the release time of VOCs. Results revealed that the pathogen fingerprint identification saved time for at least $48 \mathrm{~h}$. The relative signal intensities of the different bacterial VOCs grew at different rates, whereas the intensities of some signals remained nearly constant. The peaks at $m / z 101$ and $m / z 87$ were paid considerable attention because of their possibility as biomarker of Staphylococci; steady increase in the signal intensity in the beginning of incubation $(<12 \mathrm{~h})$ was followed by a rapid increase (Fig. 6a). However, no significant relationship with the initial concentration of bacteria was (a)

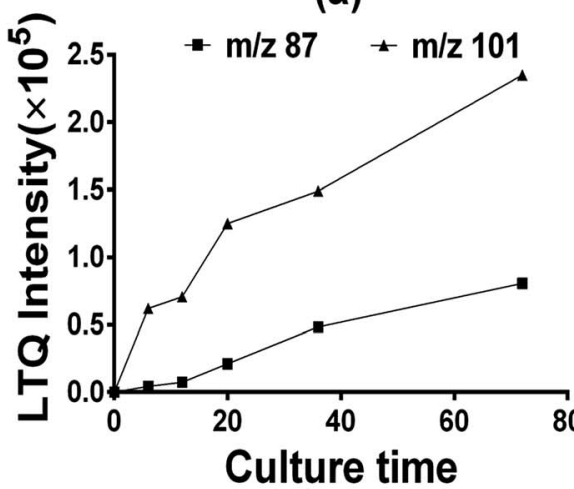

(h) (b)

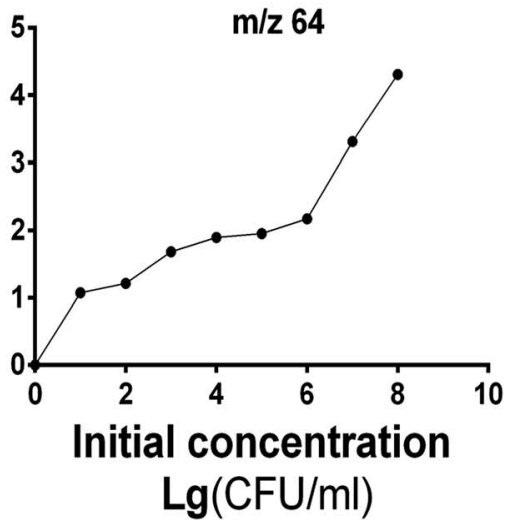

Fig. 6 The intensity of MS signals at $\mathrm{m} / \mathrm{z} 101, \mathrm{~m} / \mathrm{z} 87$ (a) and $\mathrm{m} / \mathrm{z} 64$ (b) in the VOC mass spectrum of simulated S. aureus blood cultures as a function of culture time or initial $S$. aureus concentration. 
established. The characteristic releases of isobutyric acid $(\mathrm{m} / \mathrm{z} 87$ in negative ion mode) and isovaleric acid $(\mathrm{m} / \mathrm{z} 101$ in negative ion mode) by SA cultures are respectively linked to the metabolism of valine and leucine precursor. ${ }^{\mathbf{1 3 , 1 4}}$ Therefore, the signal response of particular biomarkers can be selectively enhanced for targeted analysis by increasing the nutrient substance in culturing medium. Furthermore, signal intensity of nitrate $(\mathrm{m} / \mathrm{z} 64$ in positive ion mode) was proportional to the initial concentration of bacteria, whereas there was nothing to do with the culture time and remained constant. This appearance may be related to the ability of bacterial metabolism. There also remains space for further analytical improvement (e.g., adjustment of the medium, change of culture environment or optimization of mass spectrometry conditions). We can optimize the conditions (including $\mathrm{m} / \mathrm{z}$ range, peak intensity and peak area\%) for each $\mathrm{m} / \mathrm{z}$ peak of all the VOCs in the standard strains blood culture, then establish the fingerprint database. We need to do internal quality control with the standard strains conventionally, the establishing of cutoff value for qualitative experiment and preparation of calibrators for quantitative testing are the goal of our next phase of experimentation. The integration of this approach in clinical practice will be further promoted by the growing availability of atmospheric corona discharge ionization mass spectrometers in bioanalytical laboratories and core facilities.

\section{Conclusion}

VOCs are good biomarkers of pathogens, Staphylococci were readily distinguished based on characteristic fingerprints. The obvious advantages of CDI-MS are particularly suitable for the detection of VOCs. However, the potential of using ambient ionization approaches in real clinical practice remains largely unexplored. The results of our 6 month hospital study strongly confirmed the potential of CDI-MS for clinical applications. Therefore, Staphylococcal blood culture processed by the rapid and noninvasive CDI-MS analysis can be further interrogated by more expensive and time-consuming biochemical assays for complementary analysis.

\section{Acknowledgements}

This work is jointly supported by National Natural Science Foundation of China (NNSFC) (no. 81460327, 21105010) and major Project Science and Technology Agency of Jiangxi Province (no. 20152ACG70014).

\section{References}

1 S. K. Gohil, C. Cao, M. Phelan, T. Tjoa, C. Rhee, R. Platt and S. S. Huang, C. Centers for Disease and P. Prevention Epicenters, Clin. Infect. Dis., 2016, 62, 695-703.

2 Q. Liu, W. Li, X. Du, W. Li, T. Zhong, Y. Tang, Y. Feng, C. Tao and Y. Xie, PLoS One, 2015, 10, e0130701.

3 J. N. Wu, T. E. Gan, Y. X. Zhu, J. M. Cao, C. H. Ji, Y. H. Wu and B. Lv, J. Zhejiang Univ., Sci., B, 2015, 16, 70-77.
4 Y. Jiang, L. Kuang, H. Wang, L. Li, W. Zhou and M. Li, Intern. Med., 2016, 55, 597-603.

5 D. J. Anderson, R. W. Moehring, R. Sloane, K. E. Schmader, D. J. Weber, V. G. Fowler Jr, E. Smathers and D. J. Sexton, PLoS One, 2014, 9, e91713.

6 B. Audrain, M. A. Farag, C. M. Ryu and J. M. Ghigo, FEMS Microbiol. Rev., 2015, 39, 222-233.

7 V. Shestivska, K. Dryahina, J. Nunvar, K. Sovova, D. Elhottova, A. Nemec, D. Smith and P. Spanel, J. Breath Res., 2015, 9, 027104.

8 Y. Wang, M. Wang, J. Li, S. Yao, J. Xue, W. Zou and C. Hu, J. Antibiot., 2014, 67, 511-518.

9 R. M. Schnabel, M. L. Boumans, A. Smolinska, E. E. Stobberingh, R. Kaufmann, P. M. Roekaerts and D. C. Bergmans, Respir. Med., 2015, 109, 1454-1459.

10 C. D. Doern and S. M. Butler-Wu, J. Mol. Diagn., 2016, 18, 789-802.

11 S. P. Pasilis and G. J. Van Berkel, Encyclopedia of Spectroscopy and Spectrometry, 2017, 3rd edn, pp. 819-829.

12 S. Sethi, R. Nanda and T. Chakraborty, Clin. Microbiol. Rev., 2013, 26, 462-475.

13 A. G. James, D. Cox and K. Worrall, Flavour Fragrance J., 2013, 28, 231-237.

14 A. G. James, C. J. Austin, D. S. Cox, D. Taylor and R. Calvert, FEMS Microbiol. Ecol., 2013, 83, 527-540.

15 K. Cheng, H. Chui, L. Domish, D. Hernandez and G. Wang, Proteomics: Clin. Appl., 2016, 10, 346-357.

16 K. Sekimoto, M. Sakakura, T. Kawamukai, H. Hike, T. Shiota, F. Usui, Y. Bando and M. Takayama, Analyst, 2016, 141, 4879-4892.

17 Z. Aladaghlo, A. R. Fakhari and K. S. Hasheminasab, Microchem. J., 2016, 129, 41-48.

18 J. Liang, Y. Hang, K. Chingin, L. Hu and H. Chen, RSC Adv., 2014, 4, 25326.

19 K. Chingin, J. Liang, Y. Hang, L. Hu and H. Chen, RSC Adv., 2015, 5, 13952-13957.

20 X.-X. Li, L.-F. Chen, Y.-Z. Ouyang, F. Feng and H.-W. Chen, Chin. J. Anal. Chem., 2016, 44, 25-31.

21 H.-W. Chen, J.-H. Lai, Y.-F. Zhou, Y.-F. Huan, J.-Q. Li, Z. Xie, Z.-C. Wang and M.-B. Luo, Chin. J. Anal. Chem., 2007, 35, 1233-1240.

22 L. Hu, J. Liang, K. Chingin, Y. Hang, X. Wu and H. Chen, RSC Adv., 2016, 6, 8449-8455.

23 S. Sethi, R. Nanda and T. Chakraborty, Clin. Microbiol. Rev., 2013, 26, 462-475.

24 K. Kim, C. Mall, S. L. Taylor, S. Hitchcock, C. Zhang, H. I. Wettersten, A. D. Jones, A. Chapman and R. H. Weiss, PLoS One, 2014, 9, e86223.

25 L. D. Bos, P. J. Sterk and M. J. Schultz, PLoS Pathog., 2013, 9, e1003311.

26 C. Sales, M. I. Cervera, R. Gil, T. Portolés, E. Pitarch and J. Beltran, Food Chem., 2017, 216, 365-373.

27 J. Ding, S. Yang, D. Liang, H. Chen, Z. Wu, L. Zhang and Y. Ren, Analyst, 2009, 134, 2040-2050. 